\section{Prática esportiva e participação nas aulas de educação física: fatores associados em estudantes de Londrina, Paraná, Brasil}

\author{
Factors associated with participation in sports and \\ physical education among students from Londrina, \\ Paraná State, Brazil
}

\section{Práctica deportiva y participación en las clases de educación física: factores asociados en estudiantes de la ciudad de Londrina, Paraná, Brasil}

\begin{abstract}
This study aimed to analyze factors associated with participation in sports and physical education. The sample consisted of 827 young people selected in two stages and stratified by neighborhood of Londrina, Paraná State, Brazil. The study analyzed participation in sports and physical education in relation to socio-demographic, behavioral, and environmental variables. Factors associated with participation in physical education included: male gender, age (10-13 years), equal to high self-rated physical activity, participation in sports, moving around during classes, and having classes on the court. Factors associated with participation in sports were male gender, age (10-13years), equal to high self-rated physical activity, physical activity ( $\geq$ 7hours/week), participation in physical education, having $\geq 10$ friends, participation in sports with friends, and frequency using facilities for sports practice. These factors should be considered in programs to encourage sports and participation in physical education.
\end{abstract}

Sports; Motor Activity; Physical Education and Training
Diogo Henrique Constantino Coledam 1 Philippe Fanelli Ferraiol 1 Raymundo Pires Junior 1 Júlio Wilson dos-Santos 2 Arli Ramos de Oliveira 1

\section{Resumo}

O objetivo deste estudo foi analisar os fatores associados à prática esportiva e participação nas aulas de educação física. A amostra foi composta por 827 jovens, selecionados em dois estágios e estratificados por região da cidade de Londrina, Paraná, Brasil. Foram analisadas a prática esportiva, participação nas aulas de educação física e variáveis sociodemográficas, comportamentais e ambientais. Os fatores associados à participação nas aulas de educação física foram: sexo masculino, idade (10-13 anos), percepção de atividade física igual ou elevada, prática esportiva, movimentação durante as aulas e realizar aulas na quadra. Para a prática esportiva, os fatores associados foram o sexo masculino, idade (10-13 anos), percepção de atividade física igual/ elevada, atividade física ( $\geq 7$ horas/semana), participação nas aulas de educação física, possuir $\geq$ 10 amigos, prática de esportes com amigos e frequência de utilização de lugares para a prática. Esses fatores devem ser considerados nos programas de incentivo à prática esportiva e à participação nas aulas de educação física.

Esportes; Atividade Motora; Educação Física e Treinamento 


\section{Introdução}

A relação entre atividade física e saúde de crianças e adolescentes tem sido amplamente descrita na literatura ${ }^{1}$. Dois aspectos que contribuem para o aumento da atividade física em jovens é a prática esportiva 2 e a participação nas aulas de educação física ${ }^{3,4}$. De forma geral, ambas também apresentam relação com a saúde dos jovens, sendo que a prática esportiva está inversamente associada ao sobrepeso 5 e positivamente à saúde mental 6, enquanto que a participação nas aulas de educação física apresenta associação positiva com a aptidão cardiorrespiratória 7,8 e comportamentos saudáveis 9 . Além disso, a participação nas aulas de educação física representa um importante papel na saúde de jovens, uma vez que é inversamente associada ao sobrepeso $10 \mathrm{e}$ obesidade 11 .

Apesar da prática esportiva e da participação nas aulas de educação física estarem inter-relacionadas com a quantidade de atividade física semanal 2,3, não devem ser tratadas como sinônimos. A prática esportiva é caracterizada como a realização de jogos com a utilização de regras regulamentadas por uma federação, podendo ter como objetivo a educação, o lazer, a saúde ou o rendimento ${ }^{12}$. Com relação à aula de educação física, esta pode ser definida como disciplina obrigatória na rede básica de educação, tendo como objetivo o ensino dos jogos, esportes, atividades rítmicas, ginásticas e práticas de aptidão física em benefício da qualidade de vida ${ }^{13}$. Por fim, a atividade física é definida como qualquer movimento corporal realizado na escola, trabalho, lazer, afazeres domésticos ou deslocamentos, produzido por músculos esqueléticos que resulta em dispêndio energético acima dos níveis de repouso 14 . Sendo assim, a prática esportiva e a participação nas aulas de educação física são dois aspectos que compõem a atividade física semanal. Somadas aos outros componentes (transporte, tarefas domésticas, no trabalho, na escola e no tempo livre), devem resultar em no mínimo 300 minutos de atividade física semanal em intensidade moderada a vigorosa, recomendadas para crianças e adolescentes 15 .

Do ponto de vista epidemiológico, grande atenção tem sido dada em investigar a prevalência e os fatores associados à prática de atividade física em crianças e adolescentes brasileiros $16,17,18,19$. No entanto, há carência de informações acerca dos fatores associados à prática esportiva e participação nas aulas de educação física nessa faixa etária, o que impede a elaboração de programas de intervenção que poderiam contribuir para a diminuição da alta prevalência de jovens inativos 17. Um estudo analisou diferentes fatores sociodemográficos e comportamentais, e as únicas variáveis que apresentaram associação com a ausência nas aulas de educação física foram a idade ( $>16$ anos) e estar trabalhando 19. Apesar dos resultados encontrados, não foram analisadas as características das aulas, o que limita a generalização dos resultados obtidos e impede a análise da possível interação entre as características das aulas e a participação dos escolares. Com relação à prática esportiva, no Brasil a literatura apresenta apenas fatores motivacionais que impulsionam a prática dos jovens ${ }^{20}$. Ainda não foram investigados os fatores sociodemográficos, comportamentais e ambientais, como já descrito em outros países como Portugal, Dinamarca e Espanha 21,22,23,24.

A prevalência de participação nas aulas de educação física é de aproximadamente $50 \%$ em jovens brasileiros 19 e, apesar de ainda não ter sido descrita a prevalência de prática esportiva, em amostras de outros países foi descrito que entre $30 \%$ e $70 \%$ dos jovens praticam esportes $21,23,25$. Investigar os fatores associados torna-se relevante para identificar os jovens que possam estar em risco de serem acometidos por agravos 5,6,7,8,9,10 oriundos da não prática esportiva e não participação nas aulas de educação física. Investigar a temática em questão poderá fornecer informações relevantes para a elaboração de programas de intervenção junto à escola e comunidade, além de nortear estudos futuros com o objetivo de identificar outros fatores associados e intervenções para a promoção da prática esportiva e participação nas aulas de educação física. Por isso, o objetivo do presente trabalho foi analisar os fatores associados à prática esportiva e participação nas aulas de educação física em jovens.

\section{Métodos}

Este estudo é do tipo transversal e foi realizado na rede estadual de ensino da cidade de Londrina, Paraná, Brasil, no período de abril a julho de 2012. Nesse ano, Londrina tinha 55.475 escolares matriculados na rede estadual de ensino. O método para a seleção amostral foi o probabilístico, utilizando dois conglomerados (escola e sala de aula) e estratificado por região da cidade (norte, sul, leste, oeste e centro) e sexo, realizado em dois estágios. Inicialmente, uma escola de grande porte de cada região da cidade foi sorteada aleatoriamente, e em cada uma foi avaliada a quantidade proporcional ao número de alunos da região. Foram utilizadas salas de aulas completas, que tinham entre 20 e 35 alunos.

O cálculo do tamanho amostral necessário teve como parâmetros a população de 55.475 es- 
colares, prevalência de participação nas aulas de educação física e prática esportiva de $50 \%$, intervalo de 95\% de confiança (IC95\%), erro amostral de $5 \%$. Um número mínimo de 382 estudantes seria necessário, no entanto, devido à utilização de conglomerados na amostragem, foi adicionado o efeito de delineamento de 2 e perda amostral de $20 \%$. Foram avaliados 965 escolares, porém a amostra final foi constituída por 827 participantes ( $15 \%$ de perda amostral), uma vez que foram analisados apenas os dados dos indivíduos que preencheram todas as questões.

Todos os procedimentos foram realizados na escola onde os alunos estavam matriculados, em um único dia, por cinco pesquisadores treinados e supervisionados pelo coordenador do trabalho. Os alunos responderam a um questionário em sala de aula, com o objetivo de estimar as variáveis dependentes (prática esportiva e participação nas aulas de educação física) e as variáveis independentes (sexo, idade, número de irmãos, o responsável com quem mora, condição socioeconômica, escolaridades paterna e materna, atividade física semanal, percepção de atividade física, transporte ativo, comportamento sedentário, necessidade de muita movimentação nas aulas de educação física, local de realização destas aulas, cansaço após as aulas de educação física, número de amigos no bairro, prática esportiva com os amigos, local adequado para prática esportiva, frequência de utilização dos locais, e locais utilizados caso não haja um espaço adequado). O tempo necessário para o preenchimento do questionário variou de 30 minutos para os escolares com idades acima de 13 anos a 50 minutos para os demais.

As variáveis dependentes do estudo foram obtidas por meio de duas questões: (1) sobre a prática esportiva: "Nas atividades de lazer e de ocupação do tempo livre você pratica esportes?”, com as opções de resposta "nunca; raramente; algumas vezes; frequentemente e sempre"; e (2) participação na aula de educação física: "Neste semestre, você participou das aulas de educação física?", com as opções de resposta "sim; não".

O participante informou o sexo e a data de nascimento no questionário, a qual foi conferida consultando-se a lista de cadastro do aluno no IV Núcleo Regional de Ensino. A condição socioeconômica foi estimada por meio do Critério de Classificação Econômica Brasil (Associação Brasileira de Empresas de Pesquisa. http://www. abep.org, acessado em 12/Abr/2013), que estabelece classificações para o nível socioeconômico de acordo com a estimativa de renda média familiar: A1 (R\$ 11.480), A2 ( $\mathrm{R} \$ 8.295), \mathrm{B} 1$ (R\$ 4.754), B2 (R\$2.656), C1 (R\$ 1.459), C2 (R\$962), $\mathrm{D}(\mathrm{R} \$ 680)$ e $\mathrm{E}(\mathrm{R} \$ 415)$.
As escolaridades paterna e materna foram analisadas utilizando-se a questão: "Coloque o grau de instrução de seu pai e sua mãe.”, com as opções de resposta "analfabeto/até 3a série fundamental; 4a série fundamental; fundamental completo (8a série); médio completo (3o colegial) e superior completo".

O número de irmãos foi estimado por meio da questão de tipo aberta: “Quantos irmãos(ãs) você possui?” Para analisar com qual responsável o participante morava foi utilizada a questão: “Com quem você mora?", tendo como opções de resposta: "com meu pai e minha mãe; apenas com minha mãe; apenas com meu pai; e nenhum".

Para estimar a atividade física habitual foi utilizado o Baecke Questionnaire of Habitual Physical Activity (BQHPA) 26. No estudo foram usadas as seguintes informações: atividade física semanal, estimada por meio do cálculo do número de horas semanais em intensidade moderada a vigorosa, de atividades esportivas e/ou programas de exercícios físicos. O transporte ativo foi analisado utilizando-se a questão: "Durante quanto tempo por dia você caminha elou anda de bicicleta para ir ao trabalho, à escola e às compras?", com as opções de resposta "< 5 minutos; 5-15 minutos; 15-30 minutos; 30-45 minutos e > 45 minutos". A percepção de atividade física foi estimada por intermédio da questão: "Em comparação com outras pessoas de mesma idade, você acredita que as atividades que realiza durante seu tempo livre são fisicamente", com as opções de resposta "muito elevadas; elevadas; iguais; baixas e muito baixas".

O comportamento sedentário foi estimado por meio da questão: “Quantas horas, em média, você assiste TV, joga videogame ou usa o computador?", com as opções de resposta “< 1 hora por dia; 1 hora por dia; 2 horas por dia; 3 horas por dia; 4 horas por dia; e 5 ou mais horas por dia”. A mesma questão foi utilizada para estimar o comportamento sedentário durante a semana e nos finais de semana.

Foram investigadas três características das aulas de educação física. A necessidade de movimentação foi avaliada pela questão: "Em todas as aulas de educação física as atividades realizadas necessitaram de grande movimentação dos alunos (correr, saltar, jogar)?”, com as opções de resposta "não; sim, em algumas aulas e; sim, em todas as aulas". O local onde a aula era realizada: “Onde você realiza as aulas de educação física?”, com as opções de resposta "duas aulas na quadra; uma aula na quadra e uma na sala e; as duas na sala”. O cansaço após as aulas: “Após as aulas de educação física, você se sente cansado e sem disposição para realizar outras atividades que 
envolvam esforço (por exemplo, caminhar, correr, brincar ou jogar)?", com as opções de resposta "não; sim em apenas uma aula por semana e; sim, toda aula".

A quantidade de pessoas da mesma idade no bairro foi estimada por meio da questão de tipo aberta: "No seu bairro há quantas pessoas da sua idade?". A prática de esportes com amigos do bairro foi investigada pela questão: "Você geralmente pratica algum esporte, jogo ou realiza atividades físicas com os amigos do seu bairro?", com as opções de resposta "sim; não".

A disponibilidade de locais para a prática de atividade física próximo à residência foi analisada usando-se a questão: "No seu bairro existe algum lugar adequado (praça, parque, quadra, campo ou clube) para você jogar, brincar ou praticar algum esporte?", com as opções de resposta "não; sim, apenas um e; sim, mais que um". Os participantes informaram a frequência de utilização desses lugares: “Quantas vezes por semana você utiliza esses lugares para realizar atividades físicas, jogar ou praticar esportes?", com as respostas "nenhuma vez; apenas uma vez; duas a quatro vezes; mais de quatro vezes”. Se o participante informasse que não havia lugar próximo à residência, respondeu à questão: “Caso em seu bairro não haja praça, parque, quadra, campo ou clube, qual lugar você utiliza para jogar, brincar, praticar esportes ou realizar atividades físicas?", com as opções de resposta "rua; minha casa ou dos amigos; vou a outro bairro".

As variáveis independentes do presente trabalho foram selecionadas de acordo com as informações disponíveis sobre a associação dos aspectos sociodemográficos, comportamentais e ambientais com a prática esportiva de jovens de outros países 21,22,23,24 e com a atividade física de jovens brasileiros 16,17,18,19. As três variáveis referentes às características aulas de educação física foram incluídas no estudo com base na experiência profissional dos pesquisadores.

A reprodutibilidade do questionário utilizado no presente estudo foi testada em 70 escolares, no intervalo de sete dias. Todas as questões apresentaram reprodutibilidade aceitável, com a concordância das questões variando de $65 \%$ a $100 \%$ entre os dois momentos. Para as questões com variável numérica discreta a reprodutibilidade foi alta, com coeficiente de correlação = 0,964 $(0,926-0,983)$ para o número de irmãos e 0,852 (0,656-0,937) para o número de amigos.

A análise descritiva foi realizada usando-se a frequência absoluta e relativa das variáveis analisadas. Para testar a reprodutibilidade do questionário foi utilizado o índice kappa para as variáveis categóricas e o coeficiente de correlação intraclasse de um fator aleatório para as variáveis numéricas. Tanto na análise bivariada quanto na multivariada foi utilizada a regressão logística binária para a estimativa da razão de chance e os respectivos IC95\%. As análises foram realizadas considerando o plano amostral complexo, de acordo com o peso amostral de cada estrato e conglomerado. Na análise multivariada foi utilizada estrutura hierárquica 27 com quatro blocos de entrada das variáveis no modelo, como descrito na Figura 1. Foram incluídas nos blocos hierárquicos as variáveis que apresentaram associação de até $20 \%$ na análise bivariada, verificadas por meio do valor de p para tendência. As variáveis que apresentaram $\mathrm{p} \leq 0,05$ na análise multivariada foram consideradas significativamente associadas.

O trabalho foi aprovado pelo Comitê de Ética em Pesquisa envolvendo Seres Humanos da Universidade Estadual de Londrina sob o protocolo no 312/2011, de acordo com a Resolução no 196/96 do Conselho Nacional de Saúde. Todos os responsáveis pelos escolares que aceitaram participar do estudo assinaram o Termo de Consentimento Livre e Esclarecido, constando todos os procedimentos que seriam realizados e formas de contatos para esclarecimento de possíveis dúvidas. Ao final do estudo, os resultados foram apresentados para cada unidade escolar por meio de um relatório individual a cada escola.

\section{Resultados}

As características dos participantes do estudo são: $54,4 \%$ eram do sexo feminino, $34,7 \%$ tinham entre $10-13$ anos, 33\% de 14 a 16 anos, $53,1 \%$ eram de condição socioeconômica média, 62,7\% tinham mãe com o Ensino Médio completo, $62,4 \%$ tinham pais com o Ensino Médio completo. A maioria dos participantes morava com ambos os pais, $70,7 \%$ e $35,4 \%$ tinham apenas um irmão. Cinquenta e nove vírgula quatro por cento realizavam entre $0-2$ horas de atividade física, $28,3 \%$ entre 3-6 horas e 30,7\% tinham percepção de atividade física elevada. $\mathrm{O}$ transporte ativo entre 6-30 minutos era realizado por $51,5 \%$ dos jovens, $32,4 \%$ praticavam esportes frequentemente/sempre, $72,9 \%$ participavam das aulas de educação física, 78,6 tinham comportamento sedentário $\geq 2$ horas durante a semana e 75,6\% no fim de semana. Quarenta e quatro vírgula sete por cento relataram necessitar de muita movimentação durante todas as aulas de educação física, $55 \%$ realizavam as duas aulas na quadra e $62,3 \%$ não se sentiam cansados após as aulas. Setenta e um por cento tinham entre 1-9 amigos no bairro, 53,7\% relataram praticar esportes com amigos do bairro, 


\begin{tabular}{|l|c|c|}
\hline & Prática esportiva & Participação na aula de educação física \\
\hline Bloco 1 (variáveis demográficas) & Sexo, idade, com quem reside, número de amigos e de irmãos(ãs) \\
\hline Bloco 2 (variáveis socioeconômicas) & Condição socioeconômica, escolaridades paterna e materna \\
\hline Bloco 3 (variáveis comportamentais) & $\begin{array}{r}\text { Prática de atividade física, transporte ativo, comportamento sedentário, percepção de atividade } \\
\text { física, participação nas aulas de educação física ou prática esportiva, frequência de utilização de } \\
\text { lugares para prática esportiva e prática esportiva com amigos do bairro }\end{array}$ \\
\hline $\begin{array}{l}\text { Bloco } 4 \text { (variáveis ambientais e } \\
\text { características das aulas) }\end{array}$ & $\begin{array}{r}\text { Disponibilidade de local adequado para } \\
\text { prática esportiva no bairro e local de prática } \\
\text { caso não haja lugar disponível }\end{array}$ & $\begin{array}{c}\text { Necessidade de muita movimentação durante } \\
\text { as aulas, local de realização das aulas e } \\
\text { cansaço após a realização das aulas }\end{array}$ \\
\hline
\end{tabular}

$40 \%$ tinham mais do que um lugar para a prática esportiva no bairro, $37,1 \%$ frequentavam os lugares mais de uma vez na semana, e dentre os que não tinham lugar, $43,9 \%$ utilizavam as suas casas, $40,7 \%$ as ruas e $15,4 \%$ se deslocavam a outros bairros para praticar esportes.

Na Tabela 1 estão descritos os resultados da associação bivariada entre as variáveis independentes analisadas e a prática esportiva e participação nas aulas de educação física. Foram encontradas proporções significativamente menores de prática esportiva e participação na aula de educação física em escolares do sexo feminino e nos que tinham entre 14 e 20 anos.

Maiores proporções de prática esportiva foram encontradas entre os estudantes que tinham $\geq 10$ amigos, mãe com os ensinos Médio e Superior completos, realizavam atividade física > 3 horas/semana, tinham percepção igual ou superior às pessoas da mesma idade, realizavam transporte ativo > 30 minutos/semana, participantes das aulas de educação física, praticavam esporte com amigos do bairro, tinham mais de um local adequado à prática esportiva no bairro, utilizavam o local $\geq 1 \mathrm{vez} / \mathrm{semana}$ e, entre os que não tinham local adequado, deslocavam-se a outros bairros. Para a participação nas aulas de educação física foram encontradas maiores proporções de participação nos escolares que realizavam atividade física semanal $>3$ horas, tinham percepção de atividade física igual ou superior às pessoas da mesma idade, praticantes de esporte, que necessitavam realizar muita movimentação em algumas ou todas as aulas e nos que realizavam uma ou duas aulas na quadra. As variáveis número de irmãos, comportamento sedentário no fim de semana e cansaço após as aulas de educação física não foram descritas na Tabela 1 por não apresentarem associação com os desfechos na análise bivariada $(p>0,20)$.

A análise multivariada da associação entre as variáveis independentes com a prática esportiva e participação nas aulas de educação física são apresentados na Tabela 2. O sexo feminino e idades entre 17 e 20 anos permaneceram inversamente associados à prática esportiva e participação nas aulas de educação física. Ter entre 14 e 17 anos apresentou associação apenas com a participação nas aulas de educação física.

Maiores razões de chances para prática esportiva, que variaram de 1,81 a 3,75 vezes, foram encontradas entre os estudantes que tinham $\geq$ 10 amigos no bairro, realizavam atividade física $\geq 7$ horas/semana, tinham percepção de atividade física igual ou superior às pessoas da mesma idade, participantes das aulas de educação física, praticavam esporte com os amigos e utilizavam os locais para prática esportiva em seu bairro $\geq$ $1 \mathrm{vez} / \mathrm{semana}$. Da mesma forma, as razões de chances de participar das aulas de educação física variaram de 1,66 a 8,17 vezes superiores entre os escolares que tinham percepção de atividade física igual ou superior às pessoas da mesma idade, praticavam esporte, que necessitavam realizar muita movimentação em algumas ou todas as aulas de educação física e nos que realizavam uma ou duas aulas na quadra.

As variáveis com quem mora (bloco 1), condição socioeconômica, escolaridades materna e paterna (bloco 2), transporte ativo e comportamento sedentário (bloco 3), local adequado para a prática esportiva no bairro e em que lugar realiza a prática caso não tenha disponibilidade no bairro (bloco 4) não foram descritas na Tabela 2 
Tabela 1

Associação entre as variáveis independentes com a prática esportiva e participação nas aulas de educação física: regressão logística bivariada.

\begin{tabular}{|c|c|c|c|c|}
\hline \multirow[t]{2}{*}{ Variáveis } & \multicolumn{2}{|c|}{ Prática esportiva } & \multicolumn{2}{|c|}{ Participação nas aulas de educação física } \\
\hline & Prevalência & OR (IC95\%) & Prevalência & OR (IC95\%) \\
\hline Sexo & $p<0,001$ & & $p<0,001$ & \\
\hline Masculino & 47,7 & 1,00 & 78,4 & 1,00 \\
\hline Feminino & 19,6 & $0,25(0,18-0,34)$ & 65,7 & $0,50(0,36-0,68)$ \\
\hline Idade (anos) & $p<0,001$ & & $p<0,001$ & \\
\hline $10-13$ & 44,6 & 1,00 & 83,6 & 1,00 \\
\hline $14-16$ & 32,2 & $0,62(0,43-0,87)$ & 68,9 & $0,43(0,29-0,64)$ \\
\hline $17-20$ & 19,5 & $0,32(0,22-0,47)$ & 65,5 & $0,42(0,28-0,63)$ \\
\hline Com quem mora? & $p=0,058$ & & $p=0,619$ & \\
\hline Pai e mãe & 33,7 & 1,00 & 72,8 & 1,00 \\
\hline Apenas com o pai & 13,6 & $0,46(0,14-1,52)$ & 72,3 & $1,12(0,77-1,62)$ \\
\hline Apenas com a mãe & 31,6 & $0,85(0,59-1,21)$ & 73,8 & $1,82(0,69-4,81)$ \\
\hline Nenhum & 27,3 & $0,54(0,25-1,18)$ & 65,9 & $0,57(0,28-1,15)$ \\
\hline Número de amigos no bairro & $p=0,001$ & & & \\
\hline 0 & 26,8 & 1,00 & - & - \\
\hline$\leq 9$ & 33,2 & $1,23(0,76-1,97)$ & - & - \\
\hline$\geq 10$ & 48,0 & $2,24(1,22-4,10)$ & - & - \\
\hline Condição socioeconômica & $p=0,001$ & & $p=0,590$ & \\
\hline Baixa (C1, C2, D e E) & 30,1 & 1,00 & 75,1 & 1,00 \\
\hline Média (B1 e B2) & 32,7 & $0,79(0,56-1,11)$ & 71,6 & $0,80(0,56-1,15)$ \\
\hline Alta (A1 e A2) & 18,8 & $0,36(0,20-0,65)$ & 71,9 & $1,06(0,60-1,85)$ \\
\hline Escolaridade materna & $p=0,001$ & & $p=0,706$ & \\
\hline Fundamental incompleto & 21,3 & 1,00 & 72,3 & 1,00 \\
\hline Ensino Médio completo & 33,3 & $1,55(1,02-2,36)$ & 73,7 & $1,05(0,70-1,57)$ \\
\hline Superior completo & 41,4 & $2,10(1,27-3,50)$ & 70,0 & $0,94(0,56-1,56)$ \\
\hline Escolaridade paterna & $p=0,019$ & & $p=0,451$ & \\
\hline Fundamental incompleto & 23,9 & 1,00 & 73,0 & 1,00 \\
\hline Ensino Médio completo & 33,7 & $1,43(0,94-1,15)$ & 73,6 & $1,25(0,83-1,87)$ \\
\hline Superior completo & 38,8 & $1,63(0,97-2,71)$ & 69,0 & $0,80(0,48-1,34)$ \\
\hline Atividade física semanal (horas) & $p<0,001$ & & $p<0,001$ & \\
\hline $0-2$ & 17,3 & 1,00 & 66,6 & 1,00 \\
\hline $3-6$ & 49,1 & $4,89(3,43-6,97)$ & 79,9 & $1,88(1,30-2,73)$ \\
\hline$\geq 7$ & 66,7 & $9,12(5,62-14,79)$ & 87,3 & $2,71(1,55-4,74)$ \\
\hline Percepção de atividade física * & $p<0,001$ & & $p<0,001$ & \\
\hline Baixa/Muito baixa & 12,2 & 1,00 & 61,2 & 1,00 \\
\hline Igual & 32,6 & $3,73(2,37-5,85)$ & 75,8 & $2,08(1,44-2,99)$ \\
\hline Elevada/Muito elevada & 53,1 & $8,46(5,40-13,27)$ & 81,5 & $2,87(1,92-4,28)$ \\
\hline Transporte ativo (minutos) & $p<0,001$ & & $p=0,018$ & \\
\hline$<5$ & 25,0 & 1,00 & 68,3 & 1,00 \\
\hline $6-30$ & 28,6 & $1,15(0,49-1,75)$ & 72,3 & $1,23(0,87-1,90)$ \\
\hline$>30$ & 44,3 & $2,04(1,45-2,87)$ & 77,2 & $1,37(0,94-2,00)$ \\
\hline Participação em aulas de educação física & $p<0,001$ & & & \\
\hline Não & 17,9 & 1,00 & - & - \\
\hline Sim & 37,8 & $2,93(2,01-4,28)$ & - & - \\
\hline Prática esportiva & & & $p<0,001$ & \\
\hline Nunca/Raramente/Algumas vezes & - & - & 65,5 & 1,00 \\
\hline Frequentemente/Sempre & - & - & 84,4 & $2,93(2,01-4,28)$ \\
\hline
\end{tabular}

(continua) 
Tabela 1 (continuação)

\begin{tabular}{|c|c|c|c|c|}
\hline \multirow[t]{2}{*}{ Variáveis } & \multicolumn{2}{|c|}{ Prática esportiva } & \multicolumn{2}{|c|}{ Participação nas aulas de educação física } \\
\hline & Prevalência & OR (IC95\%) & Prevalência & OR (IC95\%) \\
\hline Comportamento sedentário semana (horas) & $p=0,001$ & & $p=0,286$ & \\
\hline$\geq 2$ & 29,6 & 1,00 & 71,5 & 1,00 \\
\hline$<2$ & 38,7 & $1,32(0,92-1,89)$ & 73,2 & $1,07(0,71-1,39)$ \\
\hline Necessidade de muita movimentação durante & & & $p<0,001$ & \\
\hline \multicolumn{5}{|l|}{ as aulas de educação física? } \\
\hline Não & - & - & 33,3 & 1,00 \\
\hline Sim, algumas aulas & - & - & 67,7 & $4,72(2,40-9,27)$ \\
\hline Sim, todas as aulas & - & - & 83,2 & $10,09(5,04-20,20)$ \\
\hline Local da aula de educação física & & & $p<0,001$ & \\
\hline As duas na sala & - & - & 40,6 & 1,00 \\
\hline Uma na quadra e uma na sala & - & - & 72,6 & $4,65(2,31-9,35)$ \\
\hline As duas aulas na quadra & - & - & 75,4 & $4,84(2,43-9,64)$ \\
\hline Prática de esportes com amigos do bairro & $p<0,001$ & & & \\
\hline Não & 20,2 & 1,00 & - & - \\
\hline Sim & 44,3 & $4,76(3,40-6,66)$ & - & - \\
\hline Local adequado para prática esportiva no bairro & $p<0,001$ & & & \\
\hline Não & 26,0 & 1,00 & - & - \\
\hline Apenas 1 & 28,7 & $1,17(0,77-1,79)$ & - & - \\
\hline Mais que 1 & 39,3 & $2,11(1,39-3,19)$ & - & - \\
\hline $\begin{array}{l}\text { Quantas vezes por semana utiliza os lugares } \\
\text { para prática esportiva? ** }\end{array}$ & $p<0,001$ & & & \\
\hline Nenhuma vez & 18,4 & 1,00 & - & - \\
\hline Apenas uma vez & 34,0 & $3,68(2,24-6,05)$ & - & - \\
\hline Mais de uma vez & 55,8 & $8,36(5,29-13,22)$ & - & - \\
\hline $\begin{array}{l}\text { Caso não tenha disponibilidade no bairro, qual } \\
\text { lugar utiliza para prática esportiva? } \star \star \star\end{array}$ & $p<0,001$ & & & \\
\hline Casa & 29,3 & 1,00 & - & - \\
\hline Rua & 33,0 & $1,82(0,76-4,38)$ & - & - \\
\hline Desloca-se a outro bairro & 54,9 & $5,29(1,76-15,89)$ & - & - \\
\hline
\end{tabular}

IC95\%: intervalo de 95\% de confiança; OR: odds ratio.

* Comparado às pessoas da mesma idade;

** Apenas escolares que relataram ter lugar para prática;

*** Apenas escolares que relataram não ter local para prática esportiva (variável que não tem relação com a variável independente analisada).

por não se manterem associadas aos desfechos na análise multivariada.

\section{Discussão}

A prevalência de jovens dos sexos masculino e feminino que praticam esportes foi de $47,7 \%$ e $19,6 \%$, e nos que participam das aulas de educação física de $78,4 \%$ e $65,7 \%$ respectivamente. A prevalência de prática esportiva foi inferior a encontrada em estudos com jovens portugueses (62\%-72\% no sexo masculino $2,21,23,34 \%-44 \%$ no feminino 2,23 ) e $60 \%$ em jovens americanos de ambos os sexos 25. Com relação à participação nas aulas de educação física, no presente estu- do foram encontradas prevalências superiores as encontradas no Brasil (50\%) 19 e Estados Unidos (60\%) 28,29.

A menor proporção de jovens do sexo feminino comparada ao sexo masculino que praticavam esporte e participavam das aulas de educação física corroboram com outros resultados já descritos na literatura 18,22,30. A diferença entre os sexos na prevalência de prática esportiva pode estar associada às experiências constrangedoras na presença do sexo oposto e imagem corporal em meninas, fazendo com as jovens optem por atividades como corrida e ginástica de academia ${ }^{29}$. Os mesmos aspectos ${ }^{29}$ podem estar influenciando a participação nas aulas de educação física, haja vista que as aulas no Brasil 
Tabela 2

Associação multivariada entre as variáveis independentes com a prática esportiva e participação nas aulas de educação física: regressão logística utilizando modelo hierárquico (Figura 1).

\begin{tabular}{|c|c|c|}
\hline Variáveis & $\begin{array}{l}\text { Prática esportiva } \\
\text { OR (IC95\%) }\end{array}$ & $\begin{array}{c}\text { Participação nas aulas de educação física } \\
\text { OR (IC95\%) }\end{array}$ \\
\hline \multicolumn{3}{|l|}{ Bloco 1} \\
\hline \multicolumn{3}{|l|}{ Sexo } \\
\hline Masculino & 1,00 & 1,00 \\
\hline Feminino & $0,27(0,18-0,40)$ & $0,53(0,38-0,72)$ \\
\hline \multicolumn{3}{|l|}{ Idade (anos) } \\
\hline $10-13$ & 1,00 & 1,00 \\
\hline $14-16$ & $0,74(0,49-1,12)$ & $0,45(0,30-0,68)$ \\
\hline $17-20$ & $0,34(0,22-0,53)$ & $0,44(0,30-0,67)$ \\
\hline \multicolumn{3}{|l|}{ Número de amigos no bairro } \\
\hline 0 & 1,00 & - \\
\hline$\leq 9$ & $1,34(0,80-2,24)$ & - \\
\hline$\geq 10$ & $2,30(1,19-4,46)$ & - \\
\hline \multicolumn{3}{|l|}{ Bloco 3} \\
\hline \multicolumn{3}{|l|}{ Atividade física seminal (horas) } \\
\hline $0-2$ & 1,00 & 1,00 \\
\hline $3-6$ & $2,51(1,55-4,07)$ & $1,11(0,71-1,71)$ \\
\hline$\geq 7$ & $3,56(1,85-6,82)$ & $1,57(0,81-3,03)$ \\
\hline \multicolumn{3}{|l|}{ Percepção de atividade física * } \\
\hline Baixa/Muito baixa & 1,00 & 1,00 \\
\hline Igual & $2,03(1,11-3,70)$ & $1,66(1,11-2,48)$ \\
\hline Elevada/Muito elevada & $3,75(2,02-6,95)$ & $1,70(1,07-2,70)$ \\
\hline \multicolumn{3}{|l|}{ Prática esportiva } \\
\hline Nunca/Raramente/Algumas vezes & - & 1,00 \\
\hline Frequentemente/Sempre & - & $1,88(1,21-2,91)$ \\
\hline \multicolumn{3}{|l|}{ Participação em aulas de educação física } \\
\hline Não & 1,00 & - \\
\hline Sim & $1,81(1,08-3,04)$ & - \\
\hline \multicolumn{3}{|c|}{ Quantas vezes por semana utiliza os lugares para prática esportiva? ** } \\
\hline Nenhuma vez & 1,00 & - \\
\hline Apenas uma vez & $2,94(1,60-5,42)$ & - \\
\hline Mais de uma vez & $3,70(2,01-6,84)$ & - \\
\hline \multicolumn{3}{|c|}{ Prática de esportes com amigos do bairro } \\
\hline Não & 1,00 & - \\
\hline Sim & $2,41(1,48-3,92)$ & - \\
\hline \multicolumn{3}{|l|}{ Bloco 4} \\
\hline \multicolumn{3}{|c|}{ Necessidade de muita movimentação durante as aulas de educação física? } \\
\hline Não & - & 1,00 \\
\hline Sim, algumas aulas & - & $3,42(1,63-7,19)$ \\
\hline Sim, todas as aulas & - & $8,17(3,71-17,97)$ \\
\hline \multicolumn{3}{|l|}{ Local da aula de educação física } \\
\hline As duas na sala & - & 1,00 \\
\hline Uma na quadra e uma na sala & - & $2,17(1,08-4,35)$ \\
\hline As duas aulas na quadra & - & $2,38(1,21-4,68)$ \\
\hline
\end{tabular}

IC95\%: intervalo de 95\% de confiança; OR: odds ratio.

* Comparado às pessoas da mesma idade;

** Apenas escolares que relataram ter lugar para prática;

Nota: variável que apresentou valor de $p>0,20$ na análise bivariada ou que não tem relação com a variável independente analisada. Variáveis que não se mantiveram associadas aos desfechos na análise multivariada não foram apresentadas. 
são realizadas em turmas mistas. Além disso, já foi descrito que jovens do sexo feminino relatam não gostar dessas aulas, provavelmente devido à menor competência atlética percebida 31 , o que reduz a participação quando comparadas aos jovens do sexo masculino 32 . Da mesma forma, outros fatores como a maior facilidade ao acesso para prática, incentivo dos amigos e prazer em realizar a atividade física podem explicar a maior prevalência de prática esportiva e participação nas aulas de educação física em jovens do sexo masculino, fatores que estão associados apenas aos rapazes 33 .

Para a idade, com o aumento dos anos ocorreu a diminuição da participação nas aulas de educação física, fato que corrobora com um estudo realizado com jovens brasileiros e que pode ser explicado pelo início do trabalho remunerado no contraturno ${ }^{19}$. Outras informações disponíveis dizem respeito apenas aos fatores que permeiam a diminuição da atividade física ao longo da idade e, apesar disto, ainda não existem resultados conclusivos sobre o tema. Um estudo longitudinal recente investigou 19 possíveis preditores da diminuição da atividade física em jovens aos 10 e 16 anos 34 . Os resultados demonstraram que nos jovens do sexo masculino apenas a atividade física moderada a vigorosa aos 10 anos e a autoeficácia estiveram associadas à atividade física, enquanto que para as jovens a atividade física moderada a vigorosa aos 10 anos e a percepção de barreiras para a prática foram associadas à atividade física 34 . Outro fator que pode estar associado à diminuição da prática esportiva ao longo da adolescência é que nesta fase começam a trabalhar e os estudos são intensificados, ocupando o tempo livre dos jovens 35,36 .

Tem sido relatado na literatura que a prática esportiva está positivamente associada à condição socioeconômica e escolaridade dos pais, sendo que este fato ocorre possivelmente devido ao suporte que crianças expostas a esta condição têm em relação a lugares e equipamentos adequados para a prática esportiva 23 . Isso já foi descrito tanto para prática esportiva quanto para atividade física vigorosa 37 . No presente trabalho os resultados são contrários à literatura, indicando que a condição socioeconômica não está associada à prática esportiva. Uma possível explicação é a não associação encontrada entre prática esportiva e disponibilidade de locais adequados para a prática. Tais resultados corroboram um estudo recente que demonstrou não haver associação entre prática esportiva e disponibilidade de locais e equipamentos adequados 21 . Esses dados permitem afirmar que possuir locais adequados não garante a prática esportiva de jovens. O que irá determinar a prática é a fre- quência do jovem nesses lugares, como descrito no presente trabalho. Dessa forma, programas de incentivo à prática esportiva devem buscar estratégias para atrair os jovens aos lugares como praças, parques, quadras poliesportivas e clubes, garantindo segurança e orientação especializada para a prática.

Outros resultados encontrados fornecem informações relevantes para o entendimento da não associação verificada entre prática esportiva e disponibilidade de locais adequados próximo à residência. Primeiro, o jovem praticante de esporte utiliza outros lugares improvisados, como por exemplo, a rua e as residências, ou ainda, se desloca para outros bairros onde há lugares adequados para praticar esportes, como verificado no presente trabalho. Além disso, quando ajustados para todas as variáveis do estudo, dois fatores, possuir $\geq 10$ amigos e praticar esportes com amigos, se mostraram positivamente associados à prática esportiva. Os resultados corroboram aos previamente descritos, os quais demonstraram que a prática esportiva está positivamente associada ao incentivo dos amigos 38 .

Da mesma forma como a prática esportiva, não foram encontradas associações entre participação nas aulas de educação física e indicadores socioeconômicos, corroborando com os resultados de um estudo realizado com jovens brasileiros 19. Nas escolas da rede estadual de ensino estão matriculados alunos de diferentes estratos socioeconômicos, como encontrado no presente estudo. Os alunos, independentemente da condição socioeconômica, usufruem da mesma infraestrutura, professores e currículo, variáveis que podem ser determinantes para a participação nas aulas de educação física quando comparadas à condição socioeconômica.

Isso foi encontrado no presente trabalho, uma vez que realizar aulas na quadra e aulas que necessitam de muita movimentação foram positivamente associadas à participação. Com relação às aulas na quadra, dois aspectos podem contribuir para maior proporção de participação nestas aulas. $\mathrm{O}$ aluno tem em média cinco aulas por dia em sala de aula com duração de 50 minutos cada, sendo assim, a aula de educação física realizada na quadra pode torná-la interessante, principalmente para os que se sentem motivados para realizar atividades físicas 39. De forma semelhante, aulas que necessitam de muita movimentação reduzem o tempo no qual o aluno permancece parado aguardando sua vez de executar tarefas ou apenas sendo instruído. A falta de movimentação nas aulas de educação física é um fator comumente relatado por alunos como sendo uma das causas da não participação ${ }^{40}$. Um fato que poderia justificar a realização de au- 
las com menor movimentação é que a aula com muita movimentação resultaria em aumento da intensidade da atividade, gerando percepções de desconforto durante a realização, o que ocasionaria a desistência da tarefa realizada, como já descrito em jovens 41 . Isso não foi confirmado pelos achados do presente estudo, haja vista que não foram encontradas associações entre se sentir cansado após as aulas de educação física e participação nas mesmas.

Um dos fatores que apresentou associação com a prática esportiva e participação nas aulas de educação física foi a percepção de atividade física. Já foi descrito que jovens superestimam a sua atividade física real ${ }^{42}$. No entanto, com relação à prática esportiva e participação nas aulas de educação física isso não ocorreu. Os resultados demonstram que indivíduos que se percebem iguais ou mais ativos fisicamente quando comparados às pessoas da mesma idade, têm maiores chances de praticar esportes e participar das aulas de educação física em relação aos que têm percepção de atividade física baixa ou muito baixa.

A atividade física apresentou associação positiva com a prática esportiva, mas o mesmo não foi encontrado para a participação nas aulas de educação física. O instrumento utilizado permitiu quantificar a atividade física realizada por meio de programas de exercício físico de qualquer tipo e prática esportiva em intensidade moderada a vigorosa. Os resultados demonstram que a prática esportiva é um aspecto importante para o acúmulo de atividade física diária semanal dos jovens brasileiros, como já relatado previamente entre jovens 2 . Assim, a prática esportiva deve ser incentivada com o objetivo de aumentar a proporção de jovens que atendem à recomendação de atividade física moderada a vigorosa semanal. Com relação às aulas de educação física, apesar de já ter sido demonstrado que elas são eficazes para aumentar a atividade física semanal de jovens 3,4 , na amostra estudada a quantidade de atividade física no tempo livre entre os que participam destas aulas não foi superior se comparada aos não participantes, como já demonstrado 19. Um fator que pode explicar esse resultado é o fato de que o incentivo e a orientação à prática de atividade física ainda não são prioridades no currículo da educação física na escola 43, característica que deve ser reavaliada tendo em vista a promoção do estilo de vida ativo dos escolares.

As duas variáveis dependentes analisadas no presente estudo apresentaram associação positiva entre si. A educação física tem como um dos seus conteúdos norteadores o esporte 13 , que além de ser praticado dentro da escola também é incentivado a ser realizado no tempo livre. Diversos são os fatores que motivam a prática esportiva de jovens, como por exemplo, promover a aptidão física e habilidades, prazer em competir e socialização 44 , itens que podem impulsionar a participação dos alunos durante as aulas apenas pelo fato de gostar de praticar esporte. Devido ao delineamento transversal utilizado no trabalho, não é possível identificar efeito causal de uma variável sobre a outra.

Apesar dos resultados encontrados, o estudo apresenta algumas limitações. Uma é o fato de que não foi analisado se o jovem realizava trabalho remunerado, variável que pode estar associada à prática esportiva. Além disso, não foram identificados quais os esportes que eram praticados pelos jovens e se o esporte era feito de forma orientada, características que podem apresentar associações diferentes das encontradas. Com relação às aulas de educação física, informações sobre a qualidade da infraestrutura, dos materiais, perfil do professor, métodos e conteúdos utilizados na aula não foram analisados, características que também podem estar associadas à participação durante estas aulas.

\section{Conclusão}

Os resultados encontrados no presente estudo sugerem que ser do sexo masculino, ter entre 10 e 13 anos e percepção de atividade física igual ou superior às pessoas da mesma idade são fatores positivamente associados com a prática esportiva e participação nas aulas de educação física. Participar dessas aulas, realizar atividade física 7 horas/semana, conviver com 10 ou mais amigos, frequentar lugares para a prática esportiva uma ou mais vezes na semana e realizar atividades com amigos estiveram positivamente associados à prática esportiva. Maiores proporções de participação nas aulas de educação física foram encontradas entre os jovens que praticam esportes, participam de aulas que necessitam de muita movimentação e nos que realizam aulas na quadra.

Os resultados encontrados apontam características que devem ser consideradas por professores e pesquisadores, tanto na execução das aulas no âmbito escolar quanto na formulação de políticas públicas para o incentivo à prática esportiva e aumento da participação nas aulas de educação física, assim como na proposta de estudos experimentais com o mesmo objetivo. Sugere-se que futuros trabalhos investiguem outros fatores, tais como conteúdos ministrados em aula e o ambiente físico e social das escolas, que também podem contribuir para o entendimento 
da participação dos jovens nas aulas de educação física. Da mesma forma, analisar fatores como barreiras ambientais, sociais e aspectos motiva- cionais torna-se relevante, uma vez que são aspectos que também podem determinar a prática esportiva de jovens.

\section{Resumen}

El objetivo de este estudio fue analizar los factores asociados a la práctica deportiva y participación en las clases de educación física. La muestra consistió en 827 jóvenes; se seleccionó en dos etapas y estratificó por región en la ciudad de Londrina, Paraná, Brasil. Se analizó la práctica deportiva, participación en las clases de educación física y variables sociodemográficas, de comportamiento y ambientales. Los factores asociados a la participación en las clases de educación física fueron: sexo masculino, edad (10-13 años), percepción de actividad física igual o superior, práctica deportiva, ejercicio en las clases y clases en pista deportiva. Para la práctica deportiva, los factores asociados fueron: sexo masculino, edad (10-13 años), percepción de actividad física igual o superior, actividad física ( $\geq 7$ horas/ semana), participación en las clases de educación física, tener $\geq 10$ amigos, práctica deportiva con amigos y frecuencia de uso de las instalaciones. Estos factores deben ser considerados en programas de incentivos a la práctica deportiva y participación en las clases de educación física.

Deportes; Actividad Motora; Educación y

Entrenamineto Físico

\section{Colaboradores}

D. H. C. Coledam, P. F. Ferraiol e R. Pires Junior atuaram na coleta, análise e interpretação dos dados, elaboração, revisão crítica e final do manuscrito. J. W. dos-Santos e A. R. Oliveira colaboraram na análise e interpretação dos dados, revisão crítica e final do manuscrito. 


\section{Referências}

1. Ekelund U, Luan J, Sherar LB, Esliger DW, Griew P Cooper A, et al. Moderate to vigorous physical activity and sedentary time and cardiometabolic risk factors in children and adolescents. JAMA 2012; 307:704-12.

2. Seabra AF, Mendonça DM, Thomis MA, Malina RM, Maia JA. Sports participation among Portuguese youth 10 to 18 years. J Phys Act Health 2007; 4:370-80.

3. Dauenhauer BD, Keating XD. The influence of physical education on physical activity levels of urban elementary students. Res Q Exerc Sport 2011; 82:512-20.

4. Meyer U, Roth R, Zahner L, Gerber M, Puder JJ, Hebestreit H, et al. Contribution of physical education to overall physical activity. Scand J Med Sci Sports 2013; 23:600-6.

5. Drake KM, Beach ML, Longacre MR, Mackenzie T, Titus LJ, Rundle AG, et al. Influence of sports, physical education, and active commuting to school on adolescent weight status. Pediatrics 2012; 130:296-304.

6. Babiss LA, Gangwisch JE. Sports participation as a protective factor against depression and suicidal ideation in adolescents as mediated by self-esteem and social support. J Dev Behav Pediatr 2009; 30:376-84.

7. Kelly IR, Phillips MA, Revels M, Ujamaa D. Contribution of the school environment to physical fitness in children and youth. J Phys Act Health 2010; 7:333-42.

8. Sanchez-Vaznaugh EV, Sánchez BN, Rosas LG, Baek J, Egerter S. Physical education policy compliance and children's physical fitness. Am J Prev Med 2012; 42:452-9.

9. Tassitano RM, Barros MV, Tenório MC, Bezerra J, Florindo AA, Reis RS. Enrollment in physical education is associated with health-related behavior among high school students. J Sch Health 2010; 80:126-33

10. Tassitano RM, Barros MVG, Tenório MCM, Bezerra J, Hallal PC. Prevalência e fatores associados ao sobrepeso e à obesidade em adolescentes, estudantes de escolas de Ensino Médio de Pernambuco, Brasil. Cad Saúde Pública 2009; 25:2639-52.

11. Cawley J, Frisvold D, Meyerhoefer C. The impact of physical education on obesity among elementary school children. J Health Econ 2013; 32:743-55.

12. Tubino MJG. Dimensões sociais do esporte. 2a Ed. São Paulo: Cortez Editora; 2001.

13. Betti M, Zuliani LR. Educação física escolar: uma proposta de diretrizes pedagógicas. Revista Mackenzie de Educação Física e Esporte 2002: 1:7381.

14. Caspersen CJ, Powell KE, Christensen GM. Physical activity, exercise, and physical fitness: definitions and distinctions for health-related research. Public Health Rep 1985; 100:126-31.

15. World Health Organization. Global recommendations on physical activity for health. Geneva: World Health Organization; 2010.
16. Farias Júnior JC, Lopes AS, Mota J, Hallal PC. Prática de atividade física e fatores associados em adolescentes no Nordeste do Brasil. Rev Saúde Pública 2012; 46:505-15.

17. Barufaldi LA, Abreu GA, Coutinho ESF, Bloch KV. Meta-analysis of the prevalence of physical inactivity among Brazilian adolescents. Cad Saúde Pública 2012; 28:1019-32.

18. Ceschini FL, Andrade DR, Oliveira LC, Araújo JF, Matsudo VKR. Prevalência de inatividade física e fatores associados em estudantes do ensino médio de escolas públicas estaduais. J Pediatr (Rio J.) 2009; 85:301-6.

19. Silva KS, Nahas MV, Peres KG, Lopes AS. Fatores associados à atividade física, comportamento sedentário e participação na educação física em estudantes do Ensino Médio em Santa Catarina, Brasil. Cad Saúde Pública 2009; 25:2187-200.

20. Krebs RJ, Santos JOL, Ramalho MHS, Nazário PFN, Nobre GC, Almeida RT. Disposição de adolescentes para a prática de esportes: um estudo orientado pela teoria bioecológica de Bronfenbrenner. Motriz Rev Educ Fís 2011; 17:195-201.

21. Autran RG, Ramos E, Pina MF, Santos MP. A associação entre a proximidade a equipamentos desportivos e a prática de atividade desportiva em adolescentes de 13 anos de idade do Município do Porto, Portugal. Cad Saúde Pública 2012; 28:549-58.

22. Gracia-Marco L, Tomas C, Vicente-Rodriguez G, Jimenez-Pavon D, Rey-Lopez JP, Ortega FB, et al. Extra-curricular participation in sports and sociodemographic factors in Spanish adolescents: the AVENA Study. J Sports Sci 2010; 28:1383-9.

23. Seabra AF, Mendonça DM, Thomis MA, Peters TJ, Maia JA. Associations between sport participation, demographic and socio-cultural factors in Portuguese children and adolescents. Eur J Public Health 2007; 18:25-30.

24. Toftegaard-Støckel J, Nielsen GA, Ibsen B, Andersen LB. Parental, socio and cultural factors associated with adolescents' sports participation in four Danish municipalities. Scand J Med Sci Sports 2011; 21:606-11.

25. Simpkins SD, Schaefer DR, Price CD, Vest AE. Adolescent friendships, BMI, and physical activity: untangling selection and influence through longitudinal social network analysis. J Res Adolesc 2013; 23:535-49.

26. Baecke JAH, Burema J, Frijters JER. A short questionnaire for the measurement of habitual physical activity in epidemiological studies. Am J Clin Nutr 1982; 36:936-42.

27. Victora CG, Huttly SR, Fuchs SC, Olinto MTA. The role of conceptual frameworks in epidemiological analysis: A hierarchical approach. Int J Epidemiol 1997; 26:224-7.

28. Diamant AL, Babey SH, Wolstein J. Adolescent physical education and physical activity in California. Policy Brief UCLA Cent Health Policy Res 2011; (PB2011-5):1-8. 
29. Johnston LD, Delva J, O'Malley PM. Sports participation and physical education in American secondary schools: current levels and racial/ethnic and socioeconomic disparities. Am J Prev Med 2007; 33(4Suppl):S195-208.

30. Slater A, Tiggemann M. Gender differences in adolescent sport participation, teasing, self-objectification and body image concerns. J Adolesc 2011; 34:455-63.

31. Cairney J, Kwan MY, Velduizen S, Hay J, Bray SR, Faught BE. Gender, perceived competence and the enjoyment of Physical Education in children: a longitudinal examination. Int J Behav Nutr Phys Act 2012; 9:26.

32. Kremer MM, Reichert FF, Hallal PC. Intensidade e duração dos esforços físicos em aulas de educação física. Rev Saúde Pública 2012; 46:320-6.

33. Wenthe PJ, Janz KF, Levy SM. Gender similarities and differences in factors associated with adolescent moderate-vigorous physical activity. Pediatr Exerc Sci 2009; 21:291-304.

34. Hearst MO, Patnode CD, Sirard JR, Farbakhsh K, Lytle LA. Multilevel predictors of adolescent physical activity: a longitudinal analysis. Int J Behav Nutr Phys Act 2012; 9:8.

35. Sherar LB, Gyurcsik NC, Humbert ML, Dyck RF, Fowler-Kerry S, Baxter-Jones AD. Activity and barriers in girls (8-16 yr) based on grade and maturity status. Med Sci Sports Exerc 2009; 41:87-95.

36. Zunft HJ, Friebe D, Seppelt B, Widhalm K, Winter AMR, Almeida MDV, et al. Perceived benefits and barriers to physical activity in a nationally representative sample in the European Union. Public Health Nutr 1999; 2:153-60.

37. Niclasen B, Petzold M, Schnohr CW. The association between high recreational physical activity and physical activity as a part of daily living in adolescents and availability of local indoor sports facilities and sports clubs. Scand J Public Health 2012; 40:614-20.
38. Johnson KE, Kubik MY, McMorris BJ. Prevalence and social-environmental correlates of sports team participation among alternative high school students. J Phys Act Health 2011; 8:606-12.

39. Aelterman N, Vansteenkiste M, Van Keer H, Van den Berghe L, De Meyer J, Haerens L. Students' objectively measured physical activity levels and engagement as a function of between-class and between-student differences in motivation toward physical education. J Sport Exerc Psychol 2012; 34:457-80.

40. Souza FTR, Pagani MM. A educação física escolar do ensino médio: a ótica do aluno. Revista Educação, Cultura e Sociedade 2012; 2:109-19.

41. Robbins LB, Pender NJ, Ronis DL, Kazanis AS, Pis MB. Physical activity, self-efficacy, and perceived exertion among adolescents. Res Nurs Health 2004; 27:435-46.

42. Corder K, van Sluijs EM, McMinn AM, Ekelund U, Cassidy A, Griffin SJ. Perception versus reality awareness of physical activity levels of British children. Am J Prev Med 2010; 38:1-8.

43. Del Vecchio FB. Atividade física e educação física escolar. Rev Bras Ativ Fís Saúde 2011; 16:78-9.

44. Sirard JR, Pfeiffer KA, Pate RR. Motivational factors associated with sports program participation in middle school students. J Adolesc Health 2006; 38:696-703.

Recebido em 18/Abr/2013

Versão final reapresentada em 29/Jul/2013

Aprovado em 29/Ago/2013 